\title{
Compliance Display using a Tilting-Plate Tactile Feedback Device
}

\author{
Seiedmuhammad Yazdian*, Andrew J. Doxon*, David E. Johnson*, Hong Z. Tan, and William R. Provancher* \\ * University of Utah, ${ }^{\ddagger}$ Purdue University
}

\begin{abstract}
This paper presents a tactile display device for replicating compliance sensation when interacting with deformable and nondeformable compliant objects in a virtual environment. Two small tilting plates approximately reproduce surface deformations of a compliant object. In addition to tactile information, kinesthetic information is rendered through a modified haptic paddle force feedback device. The tilting plates are moved in conjunction with the measured position of the user's finger as they pressed into the virtual surface. In a psychophysical experiment, we evaluated the effect of adding tilting motion of the device's actuated plates on the perceived compliance of a virtual surface with a kinesthetic stiffness of $60 \mathrm{~N} / \mathrm{mm}$. The experiment results indicate that tilting rates of 5,10 , and $20 \mathrm{deg} / \mathrm{cm}$ reduced the perceived stiffness of the surface by 3,9 , and $17 \mathrm{~N} / \mathrm{m}$, respectively. The advantages of the new device include its light-weight, low-cost, and simple design. These device features make it practical to integrate this compliance display with user interfaces for applications such as video games or even robotic surgery.
\end{abstract}

Keywords: Haptic rendering, compliance, softness, tactile information, force feedback, virtual environments.

Index Terms: H.5.2 [Information Interfaces and Presentation]: User Interfaces-Haptic I/O; J.4 [Social and Behavioral Science]: Psychology

\section{INTRODUCTION AND BACKGROUND}

Perceiving mechanical properties, such as compliance (softness), is beneficial in identification and discrimination of objects [1]. In our daily life, information about compliance helps us to accomplish tasks such as shaking hands, choosing fresh fruit [2], or estimating the pressure of a bicycle tire [3]. The sense of compliance plays a more important role in medical procedures. For example, physicians and surgeons perceive the compliance of biological tissues to diagnose symptoms of diseases [4] or to distinguish between healthy tissue and malignant tumors [5]. However, in robotic or minimally invasive surgery, this tactile information is not communicated through the rigid handles of surgical instruments [6-8]. In these circumstances, surgeons rely mostly on visual information during the operation. The provided visual information is usually limited [9] and more importantly

\footnotetext{
${ }^{*}$ Department of Mechanical Engineering,

50 S. Central Campus Drive, Salt Lake City, UT 84112, USA

${ }^{\ddagger}$ School of Electrical and Computer Engineering,

465 Northwestern Ave., West Lafayette, IN 47907, USA

muhammad.yazdian@gmail.com, adoxon@gmail.com,

dejohnso@cs.utah.edu, hongtan@purdue.edu, wil@mech.utah.edu
}

IEEE Haptics Symposium 2014

23-26 February, Houston, Tx, USA

U.S. Government work not protected by U.S. copyright cannot properly substitute for information regarding compliance, which is best perceived through the sense of touch [10]. For this reason, surgical instruments capable of sensing the mechanical properties of tissue and rendering missing tactile information to the surgeons are needed [6].

In order to properly replicate compliance, it is important to understand the principals behind its perception. Describing the contact between a user's fingerpad and a compliant object is complex. The surface deformation of the object and the fingerpad tissue is usually nonlinear [11] and viscoelastic [12]. When interacting with a deformable object, we perceive its compliance through two haptic channels - kinesthetic and tactile information [13-15]. The kinesthetic information pertains to the relationship between applied finger force and its rigid displacement (forcedisplacement characteristics can be regarded as a close correlate of kinesthetic information) [13], [15]. The tactile information is based on the relationship between the applied force and contact profile (force-area characteristics can be regarded as a close correlate of tactile information) [15-19].

Srinivasan and LaMotte [14] investigated a human tactile ability to discriminate compliance of both deformable and nondeformable surfaces. An inflated balloon and a piano key are examples of deformable and non-deformable compliant surfaces respectively. When both kinesthetic and tactile information were provided, participants could discriminate the compliance of close pairs of the specimens with high accuracy. However, in the absence of tactile information (anesthetized fingerpad) participants could not distinguish between the most compliant and least compliant deformable specimens. Similarly, when discriminating non-deformable surfaces, participants had poorer performance compared to discrimination of deformable specimens. Under passive touch conditions, participants could discriminate the compliance of deformable surfaces very well but they could not discriminate the compliance of non-deformable surfaces [14]. These findings suggest that a purely kinesthetic force feedback device cannot render the essential haptic information for discrimination of compliance without rending tactile information.

Different types of tactile display devices capable of rendering compliance information can be found in the literature. For example, Bicchi et al. designed a tactile display device using a set of telescoping cylinders to render "contact area spread rate" (CASR display) [19]. Although the cylinders are rigid, sensing the rate of change in the contact area produces artificial tactile information that is useful for compliance perception. Participants' performance in a virtual compliance recognition task using this device was close to their performance when directly touching physical specimens and better than the one provided by a purely kinesthetic device, in agreement with the findings in [14]. The concept of contact area spread rate was also used in other compliance display devices (e.g., designs by Bianchi et al. [17] and Fujita et al. [18]). 
In contrast to the CASR display, the human interfaces of the devices in [17] and [18] are flexible. The force-area characteristic and thus compliance is controlled by the amount of stretch in a fabric surface [17] or by regulating the fluid pressure beneath a rubber sheet [18]. Bianchi et al. [17] reported that the haptic perception resolution of their fabric-based device is better than that of the CASR display in [19]. In contrast to the CASR display interface, the fabric interface in [17] is seamless and flat prior to touch and has fewer moving mechanical parts, providing a more natural contact condition. The device in [18] is a purely tactile device and gross displacement of the device interface is prevented, while the device in [17] renders both tactile and kinesthetic information simultaneously.

Kimura et al. [20] also designed a device to control the contact width by wrapping a flexible surface around the users' finger. Furthermore, Kimura et al. [16] extended this concept of rendering contact width for an asymmetric contact by a 2-DOF compliance display device. Asymmetrical rendering of contact width or contact area can enhance the detection of lumps, for example tumors, via palpation. Although the tactile information provided by the device is useful for lump detection tasks, the touch sensation is not realistic due to the imposed rotation of the finger [16].

The force-area characteristics of contact are usually measured through camera and image processing information and used in real-time ([16], [17], and [20]) or mathematically modeled ([18], [19]). In order to estimate the force-area characteristics of a remote surface, the dimensions and compliance of contact area sensor should be similar to those of a human finger [16]. Kimura et al. [16] used a contact width sensor [20] and real-time processing information for estimating asymmetrical contact areas with a remote surface. The authors claimed that their contact width sensor met design requirements such that it is not sensitive to ambient light or image acquisition rate.

When rendering compliance, the kinesthetic and tactile information (force-displacement and force-area characteristics) are usually coupled. Physically deformable specimens in [14] and compliance stimuli rendered by haptic devices in [17] and [19] all provide coupled haptic information. This coupled haptic information makes it difficult to individually evaluate the contribution of each haptic modality in the perception of compliance. Tiest et al. [3], using a maximum-likelihood estimation, mathematically decoupled the contributions of each modality to be $\sim 90 \%$ tactile and $\sim 10 \%$ kinesthetic. This provides further support that most of the haptic information in compliance perception comes from the tactile information.

Using a conceptual Hertzian model for contact between a fingerpad and a compliant object, Scilingo et al. showed that both tactile and kinesthetic information are essential for the rendering of "unimodal ambiguous" objects [15]. Unimodal ambiguous objects have the same force-area characteristics but different force-displacement characteristics or vice versa. To address this need, they integrated a CASR display [19] with a Delta haptic device to render both tactile and kinesthetic information independently [15]. Interestingly, the results of their preliminary experiments indicate that compliance perception performance using pure tactile information is almost the same as that using the combined tactile and kinesthetic information.

All of the presented research emphasizes the importance of tactile information and the challenges in reproducing it, yielding complex devices with difficulties in incorporating them in practical systems. In this paper, considering the design concept used in [20], we present a simple compliance display with the goal of achieving a design that effectively communicates a broad range

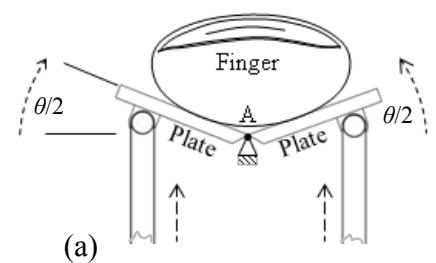

(b)

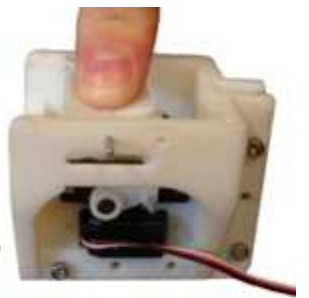

(c)

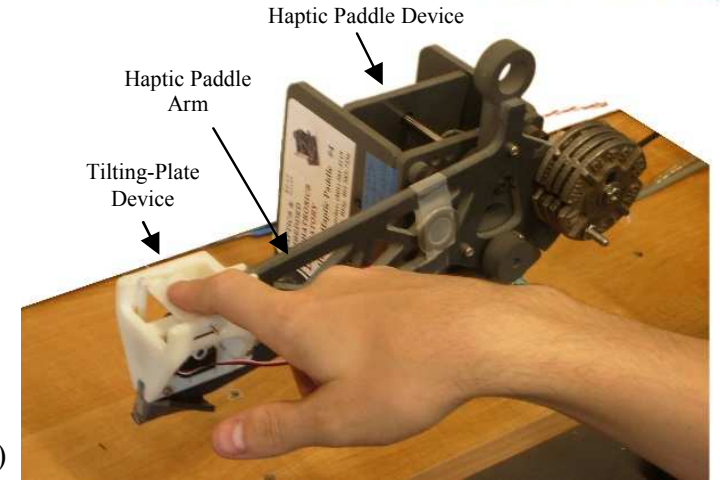

Fig. 1: The tilting-plate compliance display device. Schematic representation of device compliance display concept (a), the compliance display prototype (b), and compliance display integrated with a modified haptic paddle kinesthetic force feedback device (c).

of compliance, while also being practical to be embedded in other interfaces such as robotic surgery consoles, game controllers, or mobile devices.

\section{Device Design}

The human interface of our compliance display device consists of two small plates adjacent to each other (Fig. 1(a)). Both plates pivot about the same axis (i.e. the folding edge indicated by point A in Fig. 1(a)). The interface surface is held flat and horizontal when no compliance is rendered. When rendering compliance, the plates tilt in opposite directions to form a shallow V-shape, approximating surface deformations of a compliant object. Each small plate has a dimension of $33 \times 10 \times 2 \mathrm{~mm}$, providing a $20 \times$ $20 \mathrm{~mm}$ workspace when the plates are horizontal. Two servomotors (Futaba S3156) independently position each plate via a parallelogram four bar mechanism on each side of the device with a maximum angular velocity of about $6.3 \mathrm{rad} / \mathrm{s}$. Each tilting plate can be positioned between zero and 45 degrees with respect to the horizontal axis with an angle resolution better than 0.1 degrees. Most components of the compliance display device are rapid prototyped using PolyJet technology on an Objet 3D printer (Fig. 2(b)). The titling-plate device weight less than 55 grams.

The tilting-plate device is simple and compact. It can be mounted on kinesthetic force feedback devices, e.g. on the rotational arm of a modified haptic paddle [21] (Fig. 2(c)). This combination provides both tactile and kinesthetic information. A Maxon RE25 DC motor provides smooth motion of the rotational arm via a capstan pulley mechanism. The rotational arm is long enough $(0.2 \mathrm{~m})$ to have a negligible lateral displacement of the interface surface $(\sim 0.025 \mathrm{~mm}$ per $1.0 \mathrm{~mm}$ vertical displacement). The rotational arm is balanced by a counterweight on the other side of the arm. The rendered kinesthetic force at the center of the interface surface has a resolution of about $1 \mathrm{mN}$. This kinesthetic device can render kinesthetic stiffness levels between 20 and 500 $\mathrm{N} / \mathrm{m}$. Due to the friction and inertia of the kinesthetic haptic device, the range of compliance values that can be rendered are 
difficult to derive, especially at low and high speed motions. The entire compliance display device rotates when the rotational arm of the haptic paddle inclines. However, the two degree-of-freedom actuation of the tilting plates helps to eliminate the effect of this rotation on the plates.

A microcontroller (Microchip dsPIC33EP256MU806) receives the contact parameters (desired tilt angle of the tilting plates) via a PC computer program and commands the servomotors accordingly. The contact parameters include the amount of contact force, vertical displacement of the display, or a weighted combination of the two. The vertical displacement is measured using the haptic paddle's encoder (HEDS-5540 A02) with resolution of $\sim 0.04 \mathrm{~mm}$. For simplicity, we assumed there was a linear relationship between the displacement and motion of the tilting plates; i.e.,

$$
\theta=R \cdot d
$$

where $\theta$ is the total angle change between the plates in degrees, $R$ is the tilting rate (in $\mathrm{deg} / \mathrm{cm}$ ), and $d$ is the vertical displacement of the display in $\mathrm{cm}$. As the tilting rate increases the compliance of the rendered stimulus increases (see Section 4). The vertical displacement is also used to compute the required kinesthetic force through a linear spring model; i.e.,

$$
F=K \cdot \frac{d}{100}
$$

where $F$ is the rendered kinesthetic force in newtons and $K$ is the kinesthetic stiffness $(\mathrm{N} / \mathrm{m})$.

Also note that although each tilting plate can be controlled independently, the device only renders symmetric contact information (i.e., the tilting plates present the same contact angle to the user's fingertip). Asymmetric rendering of contact (similar to that in [16]) will not be investigated in this paper.

\section{Methods}

We hypothesized that the tactile information provided by the tilting-plate device will increase the perceived compliance (inverse of stiffness) of virtual surfaces when rendered in combination with kinesthetic feedback as compared to virtual surfaces rendered by a pure kinesthetic force feedback device. To test the effect of the tilting-plate device, we conducted a psychophysical compliance discrimination experiment.

\subsection{Participants}

A total of 10 participants ( 5 females) from the University of Utah participated in the compliance discrimination experiment. The participants' ages were between 20 and 37. All participants but one were right handed. None of the participants reported any defects in their index fingers. Before the experiment, all participants were given written instructions about the experiment and gave informed consent according to the University of Utah's IRB policy. All participants were given compensation after the experiment concluded.

\subsection{Stimuli}

The method of constant stimuli [22] was used to investigate the effect of the tactile information provided by tilting-plate on perceived compliance. The goal of the experiment was to

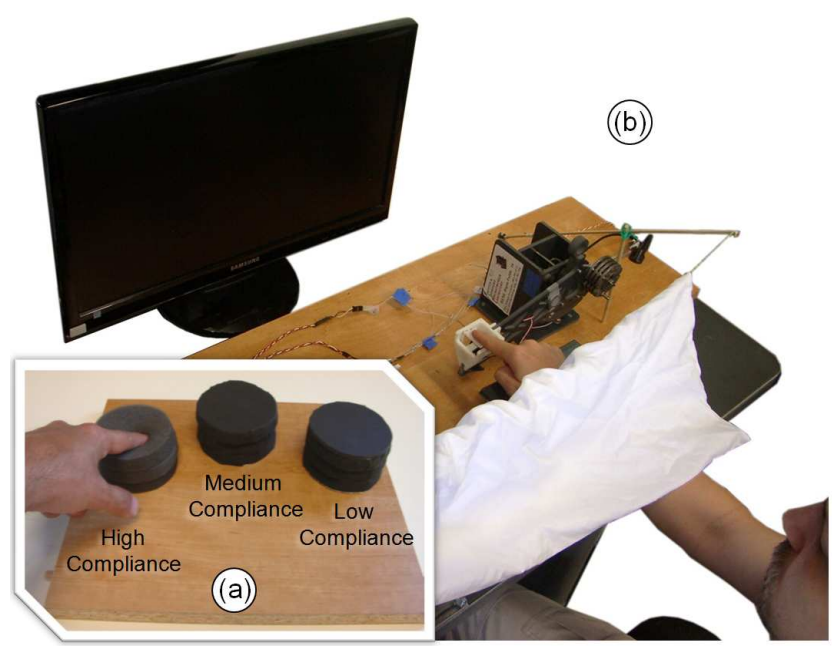

Fig. 2: Experiment setup: Three foam blocks with high, medium, and low compliance used in the training session (a). The low compliance surface is identical as the medium one but with a layer of hard foam adhered to its top. Participant performing the compliance discrimination experiment using the tiltingplate device (b). Participants could rest their hand on an arm support. Visual information regarding the motion of the device was blocked.

determine if increasing the tilting rate (the rate at which the angle between the plates changes with respect to vertical displacement of the finger) increases the perceived compliance. Reference stimuli used in this experiment included both kinesthetic and tactile contributions, with tilting rates at three different levels $(5$, 10 , and $20 \mathrm{deg} / \mathrm{cm}$ ). The kinesthetically rendered stiffness of all reference stimuli was fixed at $60 \mathrm{~N} / \mathrm{m}$. In contrast, the comparison stimuli were purely kinesthetic. A total of 9 comparison stimuli with kinesthetic stiffness levels of 20, 30, 40, 50, 60, 70, 80, 90, and $100 \mathrm{~N} / \mathrm{m}$ were used. The tilting rate levels and the kinesthetic stiffness range were determined through a pilot study and selected such that neither the plates nor the haptic paddle arm would reach their travel limits.

\subsection{Procedures}

In this experiment participants were asked to discriminate between two compliant surfaces and identify the more compliant (softer) of the two.

In a training session prior to the experiment, the participants were informed about the intended meaning of compliance (softness). In order to prevent participants from disregarding or biasing toward either of the haptic modalities, compliance was suggested to be related to the combination of surface deformation and displacement. In the training session, participants were asked to push against the surface of three physical foam blocks with high, medium, and low compliance levels and sort them based on their perceived compliance (Fig. 2(a)). The compliance levels of the blocks are chosen such that they can be distinguished easily. All three foam blocks are cylindrical with a diameter of $\sim 7.5 \mathrm{~cm}$ and a height of $\sim 4 \mathrm{~cm}$. The low compliance block was identical to the medium one but with a thin layer of hard foam adhered to its top. Thus, the kinesthetic compliance of the blocks were close to each other and they had different contact area spread rates. A semi-spherical rigid indenting tool with a $15 \mathrm{~mm}$ diameter (close to the size of a human finger) was used to measure the compliance characteristics of the foam blocks. At $1 \mathrm{~N}$ contact force level, the kinesthetic compliances of the blocks were measured to be 0.5 , 
1.0 , and $3.9 \mathrm{~mm} / \mathrm{N}$, respectively. At $1 \mathrm{~N}$ contact force level, the contact area spread rates (surface deformation characteristics) were measured to be about 45,130 , and $150 \mathrm{~mm}^{2} / \mathrm{N}$. The participants were told to push against the center of the blocks and not to slide their fingers laterally when perceiving compliance as was suggested in [23]. In the training session, the participants received correct-answer feedback on their responses.

After the training session, participants were seated at a desk and rested their wrist on an adjustable support (Fig. 2(b)). The participants were instructed to touch the surface of the device with the index finger. The position of the finger was monitored during the entire experiment to guarantee that the participant touched the two surfaces symmetrically (i.e., close to the center of the surface, where the plates meet) and perceived proper tactile information. Each stimulus was preceded by an audio cue as well as a prompt on the monitor, to indicate to the participants that they could initiate a contact with the surface. White noise was played to block any audio cues from the device. The participant's hand was obstructed by a cloth screen to prevent visual information regarding the motion of the device or the finger.

The participant's task was to perceive the compliance of two virtual surfaces and report which of the two surfaces felt more compliant using dedicated keys on a keyboard. The forced-choice design of the experiment prevented the participants from reporting that the two surfaces had the same compliance. Participants did not receive feedback on their responses and they were not allowed to retest previous surface(s) during the test. Before presenting each stimulus, participants were required to raise their finger up and break contact with the tilting plates. This allowed the device to reposition at the same initial height for all stimuli while also preventing participants from feeling a sudden change in compliance between stimuli.

The participants compared each of the three tactile-kinesthetic reference stimuli with all nine kinesthetic comparison stimuli in addition to pairs of identical comparison stimuli with kinesthetic stiffness of $60 \mathrm{~N} / \mathrm{m}$ (see Section 3.2). Each comparison was repeated 20 times for a total of 560 comparisons. Each pair of comparisons was presented in a random, but balanced order. The random order was pre-generated and was the same for all the participants. In order to reduce the effect of muscle fatigue, the actual experiment was divided in two identical sessions that were run on two different days. The entire experiment took about two hours to complete. Participants could take a break at any time during the experiment.

Prior to each session of the actual experiment, the participants performed a short series of practice comparisons using the device. The goal of the practice comparisons was to familiarize the participants with the device and the procedure of the experiment. This session was identical to the actual experiment except that only 15 practice trials were presented in a random order. No feedback was provided on responses during this practice period. The practice comparison stimuli used kinesthetic stiffness levels of $30,50,60,70$, and $100 \mathrm{~N} / \mathrm{m}$ and the reference stimuli used tilting rates of 5, 10, and $20 \mathrm{deg} / \mathrm{cm}$ and a fixed kinesthetic stiffness of $60 \mathrm{~N} / \mathrm{m}$.

\subsection{Data Analysis}

To assess the effect of our tilting-plate display on the perception of compliance, the comparison-reference pairs were scored by the percentage of trials in which the comparison stimulus (kinesthetic surface) was perceived as the more compliant surface when compared to the reference stimuli (kinesthetic + tactile). A cumulative Gaussian psychometric function [22] was fit to these scores to compute the point of subjective equality (PSE) using Probit regression in SAS. For each individual, three PSEs were obtained, corresponding to the three different tilting rates. PSEs of all participants were combined and the effect of tilting-plate device was statistically analyzed through (analysis of variance) ANOVA using SPSS.

In addition, we used the applied force and the rigid displacement metrics to investigate whether different tilting rates alter the applied force and displacement when participants interact with the compliance stimuli.

\section{Results and Discussion}

Fig. 3 shows responses for a typical participant for the different tilting-rate reference stimuli. The participant chose the comparison stimulus with the lowest kinesthetic stiffness (20 $\mathrm{N} / \mathrm{m}$ ) as the more compliant surface the majority of the time (between 90 to 100 percent) regardless of the tilting rate of the reference stimulus. Conversely, when the kinesthetic stiffness of the surface is higher than $80 \mathrm{~N} / \mathrm{m}$, the participant judged the reference stimulus as the more compliant surface (Fig. 3). From this psychometric function, the kinesthetic stiffness corresponding to the $50 \%$ response level, determines the PSE. This individual's PSEs are approximately 53, 47, and $33 \mathrm{~N} / \mathrm{m}$ for tilting rates of 5 , 10 , and $20 \mathrm{deg} / \mathrm{cm}$, respectively (see Fig. 3). This means that the compliance of a reference with a kinesthetic stiffness of $60 \mathrm{~N} / \mathrm{m}$ and tilting rate of $5 \mathrm{deg} / \mathrm{cm}$ would be perceived as the same as a flat surface (similar to what would be rendered with a typical kinesthetic display) with a kinesthetic stiffness of $53 \mathrm{~N} / \mathrm{m}$, and so on.

Fig. 4 shows the mean and $95 \%$ confidence intervals of PSEs for all participants. All three tilting rates show a significant effect on the perceived compliance/stiffness (t-tests $[\mathrm{t}(9)=27.26$, $\mathrm{p}<0.001]$ ). Increasing the tilting rate increases the perceived compliance. The mean PSEs are 57, 51, and $43 \mathrm{~N} / \mathrm{m}$ for tilting rates of 5,10 , and $20 \mathrm{deg} / \mathrm{cm}$, respectively. In other words, on average the tilting rates of 5,10 , and $20 \mathrm{deg} / \mathrm{cm}$ make the surface feel 3,9 , and $17 \mathrm{~N} / \mathrm{m}$ less stiff (more compliant) respectively, when rendered in combination with a $60 \mathrm{~N} / \mathrm{m}$ kinesthetic stiffness.

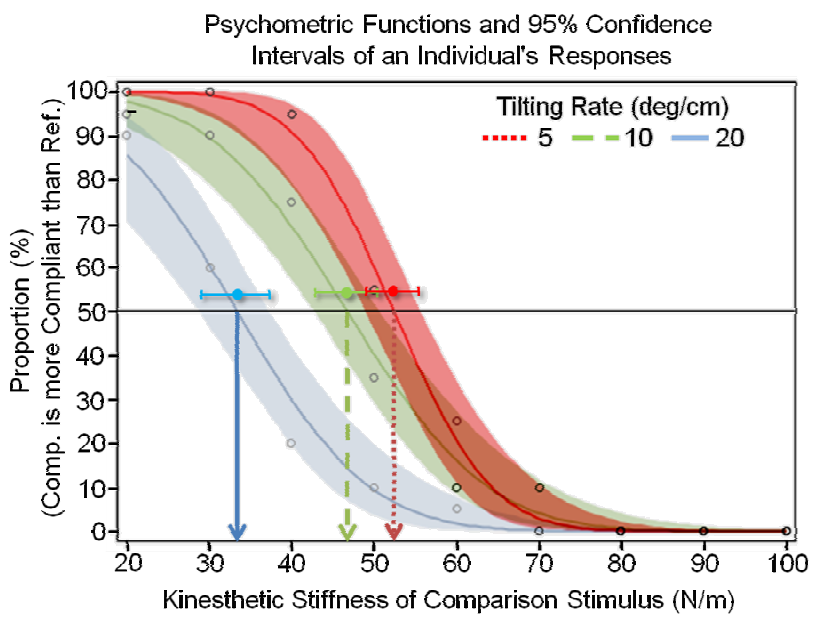

Fig. 3: Example psychometric functions for one participant with respect to reference stimuli with different tilting rates. Points of subjective equality (PSEs) for references of 5,10 and 20 $\mathrm{deg} / \mathrm{cm}$ tilting rates are approximately 53,47 , and $33 \mathrm{~N} / \mathrm{m}$. The shaded areas indicate the $95 \%$ confidence intervals. The horizontal error bars indicate the confidence intervals at $50 \%$ response level. 


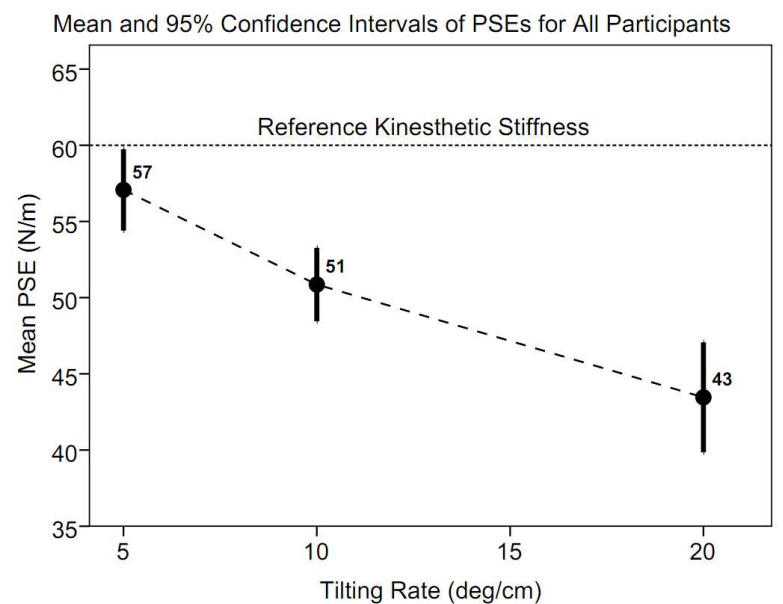

Fig. 4: Mean and $95 \%$ confidence interval of PSEs for all participants. PSEs for 5,10 and $20 \mathrm{deg} / \mathrm{cm}$ tilting rates are 57 , 51 , and $43 \mathrm{~N} / \mathrm{m}$, and are significantly different from the reference kinesthetic stiffness of $60 \mathrm{~N} / \mathrm{m}$. This means that the tilting-plate device can reduce the perceived stiffness.

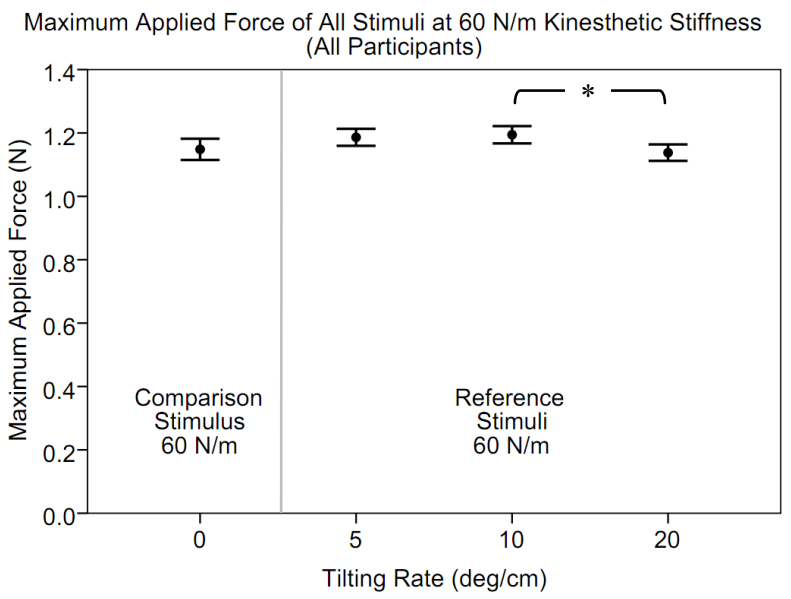

Fig. 5: Mean and 95\% confidence interval of maximum force applied to surfaces for all participants under tilting rates of zero (pure kinesthetic), 5, 10, and $20 \mathrm{deg} / \mathrm{cm}$. The kinesthetic stiffness of all surfaces is $60 \mathrm{~N} / \mathrm{m}$. No significant effect on force was found due to adding tilting plates. However, for the reference stimuli, participants applied significantly less force with a tilting rate of $20 \mathrm{deg} / \mathrm{cm}$ compared to their applied force for stimuli with a tilting rate of $10 \mathrm{deg} / \mathrm{cm}$.

For kinesthetic stiffness levels other than $60 \mathrm{~N} / \mathrm{m}$, the effect of the tilting-plate feedback is similar, but may be slightly different. Based on our pilot studies, we hypothesize that as the kinesthetic stiffness of the reference surface increases, the effect of tilting rate on perceived compliance increases. In other words, when the kinesthetic stiffness is high, we expect the addition of tilting-plate feedback to have a greater relative effect on perceived compliance.

We also investigated the maximum applied force when different tilting rates were rendered. Since the kinesthetic stiffness of all references is $60 \mathrm{~N} / \mathrm{m}$, the force applied to the reference surfaces can be directly compared against the force applied to the comparison surface with a kinesthetic stiffness of $60 \mathrm{~N} / \mathrm{m}$ (and no tilting). An ANOVA with a multiple comparison post-hoc Dunnett's T3 test (to account for unequal variances due to fewer
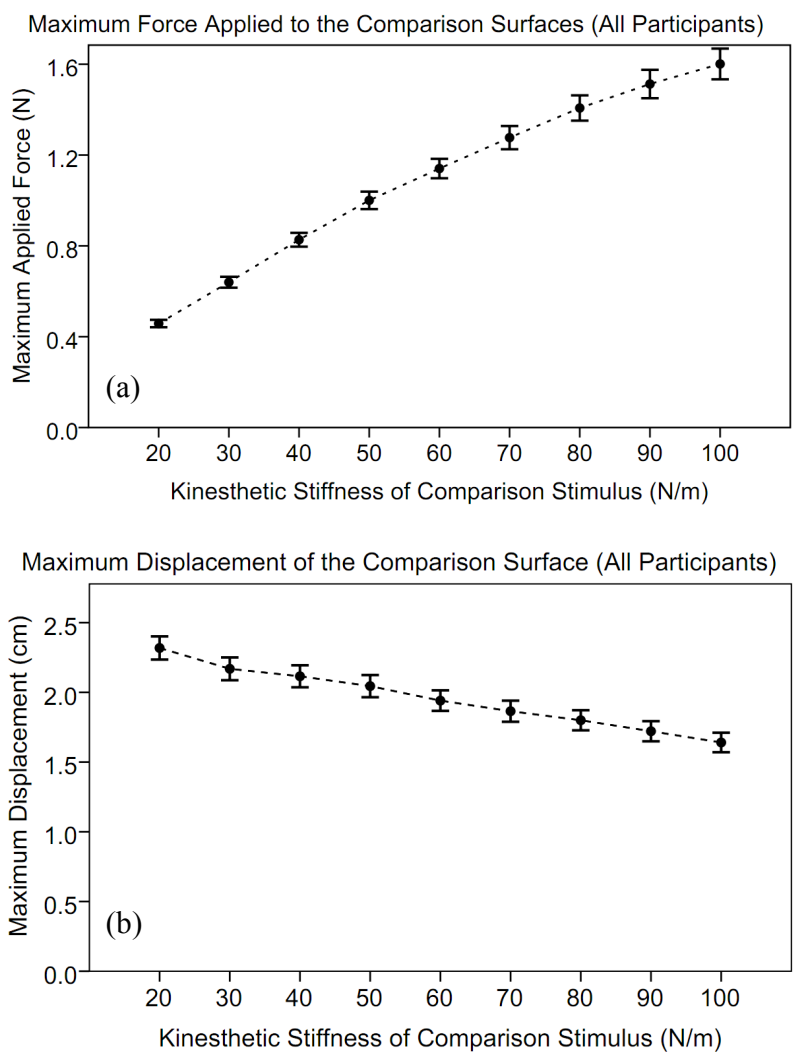

Fig. 6: Mean and 95\% confidence interval of all participants for maximum force applied to the comparison surface (a). Mean and $95 \%$ confidence interval of all participants for maximum displacement of the comparison surface (b). As kinesthetic stiffness increases, applied force increases while displacement decreases. This shows participants do not judge compliance of a non-deformable surface through a fixed force or fixed displacement across stiffness levels.

data points in the zero tilting rate group) was performed on the maximum applied forces (see also Fig. 5). The analysis of variance (ANOVA) revealed a significant difference among the applied forces $[F(3,6396)=3.94, p=0.01]$. On average, participants applied less force with a tilting rate of $20 \mathrm{deg} / \mathrm{cm}$ compared to with a tilting rate of $10 \mathrm{deg} / \mathrm{cm}$ (Dunnett's T3 test $p=0.02)($ Fig. 5). This difference may indicate that the tactile information provided by the tilting-plate device helped participants to judge the compliance with less force. However, the level of maximum applied forces under 5, 10, and $20 \mathrm{deg} / \mathrm{cm}$ tilting rate conditions were not found to be statistically different from that under the zero tilting rate (pure kinesthetic) condition. Based on the discussion that follows, it is more likely that the participants applied roughly the same amount of force across the tilting rates. From Eqn. (2), one could also conclude that the maximum displacement for judging the compliance stimuli was similar across the tilting rates $(\sim 2 \mathrm{~cm})$.

Note that the maximum force applied and the maximum displacement depend more on the kinesthetic stiffness than on the tilting rate (Fig. 6(a) and (b)). As the kinesthetic stiffness increases, maximum force applied to the comparison surface increases while displacement decreases. This shows that the participants do not judge compliance (softness) of a nondeformable surface through a fixed force or a fixed displacement across stiffness levels. 


\section{Conclusions}

We designed a simple and compact tactile display device to replicate compliance (softness) sensation of both deformable and non-deformable objects. This device can enhance the haptic rendering of compliance in virtual or teleoperated environments. The interface of the device consists of two small tilting plates that can approximate a deforming surface as force is applied to the interface. The rate of tilting is a function of finger displacement (or contact force). We integrated the tilting-plate compliance display device with a kinesthetic force feedback device (haptic paddle) to render both haptic modalities simultaneously. To determine the effect of the tilting-plate display on the perceived compliance, a psychophysical experiment was conducted. Participants discriminated the compliance of a kinesthetically rendered surface versus a kinesthetically and tactilely rendered surface using our device. The results indicate that increasing the tilting rate per unit of finger displacement makes the surface feel more compliant, hence validating our hypothesis. Tilting rates of 5, 10, and $20 \mathrm{deg} / \mathrm{cm}$ reduced the perceived stiffness of the surface by 3,9 , and $17 \mathrm{~N} / \mathrm{m}$ respectively (i.e., about $1 \mathrm{~N} / \mathrm{m}$ reduction in perceived stiffness per unit change in the tilting rate, in $\mathrm{deg} / \mathrm{cm})$. On average, at the same level of the kinesthetic stiffness $(60 \mathrm{~N} / \mathrm{m})$, participants applied similar amount of force to a deformable surface (with tilting-plate feedback) and a nondeformable surface (pure kinesthetic).

Since this tilting-plate device is simple, low-cost and lightweight, it can be easily integrated with a large range of human interfaces for applications such as virtual reality, laparoscopic surgery, video games, or mobile interfaces.

\section{FUtURE WORK}

To further investigate the effect of tilting-plate feedback, more psychophysical experiments will be conducted under different levels of reference kinesthetic stiffness. The current tactile device can also be attached to a force sensor to isolate the effect of tilting-plate feedback from kinesthetic information contributing in the compliance perception. We are interested in investigating if this stand-alone device could be a substitute for large kinesthetic force feedback devices when rendering compliance, and also evaluating the effect of tilting-plate feedback in a pinch grasp configuration.

\section{ACKNOWLEDGMENT}

This work was supported, in part, by the US National Science Foundation under award IIS-0904456 and IIS-0904403.

\section{REFERENCES}

[1] R. Klatzky and S. Lederman, "Touch," in A. Healy and R. Proctor (Eds.), Experimental Psychology (pp. 147-176), in Handbook of Psychology, vol. 4, Weiner (Ed.), New York: Wiley, 2002.

[2] B. Greer, "A guide to buying fresh fruit and vegetables," The University of Tennessee Institute of Agriculture. [Online]. Available: https://utextension.tennessee.edu/publications/Documents/SP527.pdf

[3] W. Bergmann Tiest and A. Kappers, "Cues for haptic perception of compliance," IEEE Transactions on Haptics, vol. 2, no. 4, pp. 189 $199,2009$.

[4] J. Klaesner, M. Hastings, D. Zou, C. Lewis, and M. Mueller, "Plantar tissue stiffness in patients with diabetes mellitus and peripheral neuropathy," Archives of Physical Medicine and Rehabilitation, vol. 83, no. 12, pp. 1796-801, Dec. 2002.

[5] M. Barton, R. Harris, and S. Fletcher, "Does this patient have breast cancer?," JAMA: the Journal of the American Medical Association, vol. 282, no. 13, pp. 1270-1280, Oct. 1999.
[6] P. Culmer, J. Barrie, R. Hewson, M. Levesley, M. Mon-Williams, D. Jayne, and A. Neville, "Reviewing the technological challenges associated with the development of a laparoscopic palpation device," International Journal of Medical Robotics and Computer Assisted Surgery, vol. 8, no. 2, pp. 146-159, 2012.

[7] A. Bicchi, G. Canepa, D. De Rossi, P. Iacconi, and E. Scillingo, "A sensor-based minimally invasive surgery tool for detecting tissue elastic properties," IEEE International Conference on Robotics and Automation, vol. 1, pp. 884-888, 1996.

[8] A. Okamura, "Haptic feedback in robot-assisted minimally invasive surgery," Current Opinion in Urology, vol. 19, no. 1, pp. 102-107, 2009.

[9] L. Haveran, Y. Novitsky, D. Czerniach, G. Kaban, M. Taylor, K. Gallagher-Dorval, R. Schmidt, J. Kelly, and D. Litwin, "Optimizing laparoscopic task efficiency: The role of camera and monitor positions," Surgical Endoscopy and Other Interventional Techniques, vol. 21, no. 6, pp. 980-984, 2007.

[10] R. Klatzky, S. Lederman, and D. Matula, "Haptic exploration in the presence of vision," Journal of Experimental Psychology: Human Perception and Performance, vol. 19, no. 4, pp. 726-743, 1993.

[11] K. Johnson, Contact Mechanics. Cambridge, U.K.: Cambridge Univ. Press, 1985.

[12] D. Pawluk and R. Howe, "Dynamic lumped element response of the human fingerpad," Journal of Biomechanical Engineering, vol. 121, no. 2 , pp. 178-183, 1999.

[13] W. Tiest and A. Kappers, "Kinaesthetic and cutaneous contributions to the perception of compressibility," in Haptics: Perception, Devices and Scenarios, vol. 5024, M. Ferre (Ed.), Springer Berlin Heidelberg, pp. 255-264, 2008.

[14] M. Srinivasan and R. LaMotte, "Tactual discrimination of softness.," Journal of Neurophysiology, vol. 73, no. 1, pp. 88-101, 1995.

[15] E. Scilingo, M. Bianchi, G. Grioli, and A. Bicchi, "Rendering softness: Integration of kinesthetic and cutaneous information in a haptic device," IEEE Transactions on Haptics, vol. 3, no. 2, pp. 109-118, 2010.

[16] F. Kimura, A. Yamamoto, and T. Higuchi, "Development of a 2DOF softness feeling display for tactile tele-presentation of deformable surfaces," IEEE International Conference on Robotics and Automation, pp. 1822-1827, May 2010.

[17] M. Bianchi, A. Serio, E. Scilingo, and A. Bicchi, "A new fabricbased softness display," IEEE Haptics Symposium, pp. 105-112, 2010.

[18] K. Fujita and H. Ohmori, "A new softness display interface by dynamic fingertip contact area control," The 5th World Multiconference on Systemics, Cybernetics and Informatics, pp. 7882, 2001.

[19] A. Bicchi, E. Scilingo, and D. De Rossi, "Haptic discrimination of softness in teleoperation: the role of the contact area spread rate," IEEE Transactions on Robotics and Automation, vol. 16, no. 5, pp. 496-504, 2000.

[20] F. Kimura, A. Yamamoto, and T. Higuchi, "Development of a contact width sensor for tactile tele-presentation of softness," The 18th IEEE International Symposium on Robot and Human Interactive Communication, pp. 34-39, Sep. 2009.

[21] W. Provancher and A. Doxon, "University of Utah Haptic Paddle." [Online]. Available: http://eduhaptics.org/index.php/HapticDevices/ HapticPaddles.

[22] G. Gescheider, Psycophysics: The Fundamentals, 3rd ed. London: Associates, Lawrence Erlbaum, 1997.

[23] S. Lederman and R. Klatzky, "Relative availability of surface and object properties during early haptic processing.," Journal of Experimental Psychology: Human Perception and Performance, vol. 23 , no. 6 , pp. $1680-1707,1997$. 